\title{
Approximation of Large Games with Applications to Uniform Price Auctions
}

\author{
Aaron Bodoh-Creed \\ Cornell University, Dept. of Economics, \\ 462 Uris Hall, \\ Ithaca, NY 14853 \\ acreed@cornell.edu
}

\begin{abstract}
We provide a framework justifying the use of nonatomic limit model approximations to analyze the large market behavior of otherwise intractable game-theoretic models. We demonstrate continuity requirements on the economic primitives sufficient for the equilibrium strategies of the two models to converge as the number of participants in the large finite game approaches infinity. We apply our analysis framework to show that the equilibrium of a large interdependent values uniform price auction model where bidders have complementary preferences for multiple units can be approximated by a nonatomic exchange economy. We prove that the uniform price auction asymptotically aggregates idiosyncratic bidder information into market price and that the uniform price auction is approximately efficient with a large number of participants in the private values or single unit demand case.
\end{abstract}

Keywords: Approximate Equilibrium, Large Games, Uniform Price Auction, Rational Expectations Equilibrium. JEL Codes: C72, D44, D5. 